MATHEMATICAL ASSOCIATION

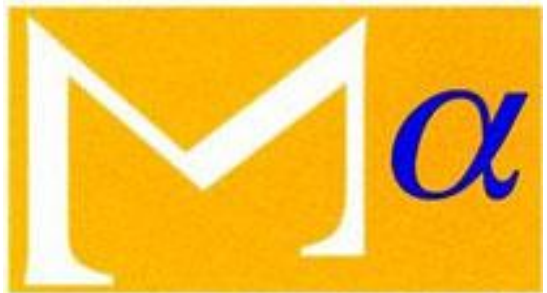

supporting mathematics in education

Analysis and Projective Geometry

Author(s): C. V. Durell

Source: The Mathematical Gazette, Vol. 6, No. 92 (May, 1911), pp. 84-87

Published by: Mathematical Association

Stable URL: http://www.jstor.org/stable/3603348

Accessed: 21-11-2015 04:15 UTC

Your use of the JSTOR archive indicates your acceptance of the Terms \& Conditions of Use, available at http://www.jstor.org/page/ info/about/policies/terms.jsp

JSTOR is a not-for-profit service that helps scholars, researchers, and students discover, use, and build upon a wide range of content in a trusted digital archive. We use information technology and tools to increase productivity and facilitate new forms of scholarship. For more information about JSTOR, please contact support@jstor.org. 
whence it is plain that all the members of the above sequence are less than 2000 , however far we like to go. It is therefore impossible by this method of procedure for the inhabitants of that community ever to reach $£ 3000$.

1 hope to deal with the Teaching of Convergent Sequences in a subsequent paper, and meanwhile beg to express my indebtedness to Dr. Charles M'Leod, Aberdeen Grammar School, for criticising the present article while in manuscript form.

W. P. Milne.

\section{ANALYSIS AND PROJECTIVE GEOMETRY.}

Few branches of elementary mathematics have escaped reform in recent years; algebra, trigonometry, elementary geometry, statics, and the calculus have all been transformed to suit the practical needs of the time. But amid all this change, there have been few attempts to alter the methods employed in introducing students to the geometry of the conic. Dr. Filon, in his most interesting treatise on Projective Geometry published in 1909 , points out that students learn the same facts about the conic practically three times over, (1) analytically, (2) through a course of what is called "geometrical conics" based on the focus-directrix definition, and (3) projectively; and his book indicates a method of co-ordinating and uniting the last two systems of approach. Although it is undoubtedly the general custom in this country to start in the first place from the focus definition, it is worth while considering afresh whether this plan is really the most advantageous.

It is interesting to note that historically this was not the starting point of the early geometers. It is true that the focus-directrix property was known to Pappus, but so far from being regarded as fundamental, it was actually lost sight of altogether, until attention was called to it by Newton. The geometrical investigations of Apollonius were based on the conception of the conic as the plane section of a cone, the advantages of which plan may be enumerated roughly as follows:

In the first place, by the aid of a model it is easy to show the shapes of the different species of conics that arise, the nature of the transition from one to the other, and their relation to and dependence upon the circle. And this emphasises the sense of continuity which should link up the student's knowledge of circle-properties to the corresponding generalised conic-properties, and enables him to extend to the conic with valuable rapidity a whole class of properties of the circle, with which he is already familiar. To take a simple example, the existence of a diameter of a conic seems a mere accident when evolved from the focus definition, but under a projective treatment such an idea, so far from appearing a happy chance, is seen to be part of the logical harmony of the subject-and this with still greater clearness, if by the aid of analysis the methods of orthogonal projection are introduced at the start.

And then, again, a factor of considerable importance is the novelty of idea with which the student is faced. The ordinary course of geometrical conics provides excellent practice in rider work, and may well supply a systematic acquaintance with the metrical theorems, but the methods employed are those to which he is already accustomed, and few, if any, of the ideas it yields are of a new character. Whereas the projective treatment opens out to view a region of geometrical thought unlike anything that has been met with in the past, and so not only arouses interest, but also may be justly regarded as of real educational value. The conception of imaginary elements and the proper appreciation of the meaning of ideal points or points at infinity and the 
applications of general projection are stimulating and fruitful; and, at the same time, the student's vanity is flattered by the gift of a weapon which enables him to discover and invent all kinds of complicated properties for himself.

On all these grounds it may be urged that projective methods should be introduced at as early a stage as is practicable. But, of course, it may be said that the difficulties and novelties of the subject require a definite maturity of mind which is only attained by students towards the end of their school course. The object of this paper is to argue that a judicious intermixture of geometrical and analytical methods will do much to smooth the path and enable the subject to be started as soon as a simple acquaintance with the analytical geometry of the circle and its geometrical harmonic properties has been attained, and before the formal course of geometrical conics is taken. It is therefore desirable to summarise the reasons why it is advantageous to employ analysis from the start.

By building upon an analytical foundation, the introduction of imaginary and ideal elements follows with natural ease from the elementary principles of algebra. The idea, for example, that any line meets any circle at two points, real or imaginary, conveys no meaning to the student, unless he has in his mind an analytical definition of what. constitutes a circle; but, as soon as he looks at it through analytical glasses, it presents no difficulties; and the first step has been taken towards an understanding of the true bearing of the Principle of Continuity. For the average student, this Principle must be merely an interpretation of an analytical phenomenon, and such applications of it, as are made, are most easily grasped if he keeps the analytical aspect always in front of him. Otherwise there is a very real danger that it never becomes more than a vague and undefined privilege, which appears to be claimed for the purpose of abbreviating proofs of certain general theorems.

The desirability of expounding the principles of general projection, for which imaginary elements are required, has been already noticed. No one can help being interested by the peculiar properties of the circular points and isotropic lines. There is so much that at first sight seems anomalous, the absence of any sense of direction and curious metrical effects all combine to cause both amusement and curiosity. And analytical methods are peculiarly suited for the investigation of these phenomena.

But another consideration of greater importance is the fact that if analysis is discarded, it is necessary to postpone the treatment of imaginary projection until after a study of involution. Prof. Lodge, at the general meeting of the Mathematical Association in 1909, pointed out the advisability of introducing a simple course on involution at an early stage. Whether or not this is adopted, it is at least probable that analytical methods would still further elucidate the difficulties of the subject. But in any case the definition of a conjugate point-pair as the double points of an overlapping involution is likely to give an air of artificiality to the whole theory, and to convey, at any rate to the average student, no meaning whatever. And this all combines to convince him that the subject is little more than a complicated conjuring-trick, which he will never be able to perform himself. But a still further disadvantage lies in the fact that the theory of foci must also be postponed till after a course of involution, or else must be developed (projectively) in a one-sided manner, which is out of keeping with the other broad conceptions of the subject. The definition of the foci as the meets of isotropic tangents is so fruitful a view-point, and may be made the base 
of so symmetrical a development, that it is most desirable that it should be introduced at the earliest opportunity.

The purport of this argument will be rendered clearer by the addition of a few details as to an analytical treatment of the kind mentioned above, which has been in use for a few vears.

To start with, it is necessary to provide definitions which cover the case of imaginary elements, such as the point, line, angle and cross-ratio : and for this object, it is well to take the analytical results, proved for real elements, and embody them in a definition for imaginary elements, e.g. the cross-ratio of the lines $y=m_{1} x, y=m_{2} x, y=m_{3} x, y=m_{4} x$, some of which are imaginary, would be defined as $\frac{\left(m_{1}-m_{2}\right)\left(m_{3}-m_{4}\right)}{\left(m_{1}-m_{4}\right)\left(m_{3}-m_{2}\right)}$, etc. It is then easy to prove a number of simple but important geometrical properties, as for example Laguerre's theorem that if $\alpha$ is the angle between the lines $y=m x, y=m^{\prime} x$, then the cross-ratio of the four lines $y=m x, y=i x, y=m^{\prime} x, y=-i x$ is $\exp (2 \alpha i)$, and deduce several important results, such as the fact that any pair of perpendicular lines are harmonically conjugate to the isotropic lines.

The next step is to introduce the conception of ideal points. If this is done by identifying every system of parallel lines with an ideal point and the aggregate of such points with the line at infinity, an analytical representation may then be effected as follows:

Take $\frac{x}{z}$ and $\frac{y}{z}$ as the Cartesian coordinates of a point, where $x, y, z$ are connected by the invariable relation $x+y+z=1$; by considering the equations of two or more parallel lines, it then appears that $z=0$ is to be regarded as representing the line at infinity and it follows immediately that any circle $x^{2}+y^{2}+2 g x z+2 f y z+c z^{2}=0$ meets this line at two points, $\omega, \omega^{\prime}$ say, which are the same for all circles and that any curve of the second degree through $\omega, \omega^{\prime}$ must be a circle. It is scarcely necessary to add that a considerable anount of general discussion is needed before the class understands the real significance of these points and their hypothetical nature.

To open the way to the conic, it is next necessary to obtain a relation between the coordinates of a point and those of its conical projection.

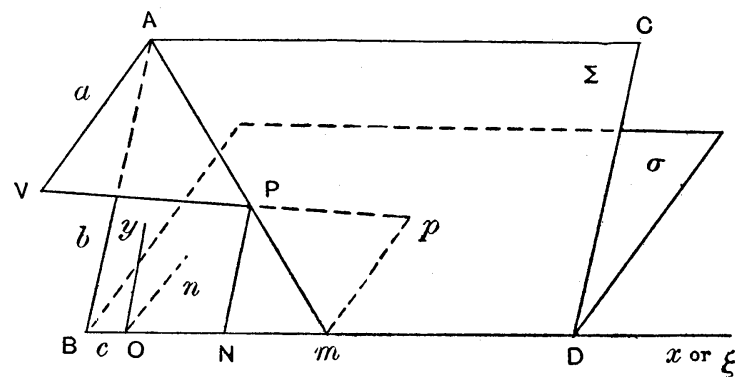

Let $P$ be any point in the plane $\Sigma$ and let $p$ be its projection on the plane $\sigma$ with respect to the vertex of projection $V ; B D$ is the line of intersection of $\Sigma$ and $\sigma$; a plane through $V$, parallel to $\sigma$, meets $\Sigma$ in the line $A C$; a plane through $V$ perpendicular to $B D$ meets $A C, B D$ in $A, B ; A P$ meets $B D$ in $m$ and the perpendicular from $P$ to $B D$ meets it in $N$. Any point $O$ on $B D$ is taken as origin, and $O D$ is the $x$-axis for $\Sigma$ and the $\xi$-axis for $\sigma$, and the coordinates of $P$ and $p$ referred to these systems of axes are respectively $x, y$ and $\xi, \eta$. The position of $V$ is determined by the following constants : $V A=a, A B=b, B O=c$. By using the similar triangles 
$V A P, p m P$ and the fact that $P N, A B$ are parallel, it is easy to show that

$$
x=\frac{a \xi-c \eta}{\eta+a}, \quad y=\frac{b \eta}{\eta+a} .
$$

It is of course obvious that this relation provides short proofs of a number of projection-theorems and may be used to establish rigorously the validity of imaginary projection. It is easy to find for instance a simple transformation of this kiud which turns the general equation of the second degree into that of a circle, or one which will project any two given points into $\omega, \omega^{\prime} ;$ for example, the relation $\xi=\frac{g x}{y-g}, \eta=\frac{i f y}{y-g}$ projects the points
$(f, g),(-f, g)$ into $\omega, \omega^{\prime}$.

In the final stage, after defining the conic as the projection of a circle and giving the usual projective definitions of the centre, asymptotes, parabola, etc., it may then be shown that every conic is represented by an equation of the second degree and that every conic through $\omega, \omega^{\prime}$ is a circle and that $C \omega, C \omega^{\prime}$ are the asymptotes of any circle, centre $C$. The foci are then defined as the meets of the tangents from $\omega, \omega^{\prime}$ to the conic and their properties and also those of confocal conics follow easily from the harmonic properties of the circumscribing quadrilateral.

Although no doubt a considerable amount of rider-work will be essential, this course now leaves the student in the position of being able to use to the fullest degree every form and variation of general projection. And by its aid, many of the more complicated theorems which still lie before him may be reduced to simpler terms and attacked with success. But it should be noted that the extent of previous reading which is required is really of quite a limited character. If, at every opportunity that presents itself, the student is encouraged to compare geometrical and analytical processes, his appreciation of the significance of the properties he is investigating will be deepened and his resources will be strengthened. Each line of attack is adapted to a special range of circumstance and each has its peculiar needs which may be supplied by the other.

C. V. Durell.

\section{CORRESPONDENCE.}

\section{To THE EdTor of THE Mathematical Gazette.}

SiR,-We have now had approximately ten years' experience of the modern - as opposed to Euclidean-methods of teaching geometry. After such a lapse of time it is only business-like to review the situation.

It is not here my purpose to question the general success of the reform, about which there can be no doubt, but rather to plead for the discussion of a small but vital portion of the subject.

My contention is, reactionary as it may appear, that the absolute elements of geometry should be taught according to one recognised order. This, it will be said, would destroy at a stroke the liberty and elasticity of the present methods; but in the preliminary work there can be no such freedom for the individual master ; in an average school the mathematical authorities fix the sequence to be followed, and master and boy must conform to it. There is within my knowledge one school where the contrary practice obtained; the beginners in the lower classes encountered no less than three different orders, but the results were not such as to commend the experiment. Apart from this instance, have we not all suffered from and pitied the boy, who, after learning according to one order at a preparatory school, is condenmed to follow another later on?

Surely this so-called elasticity, when practised on the beginner, can end in nothing but a truly British muddle, bad alike for the pupil, for his teachers, 